\title{
INTENSITY MAPPING OF MOLECULAR GAS DURING COSMIC REIONIZATION
}

\author{
C. L. CARILli \\ National Radio Astronomy Observatory, P.O. Box 0, Socorro, NM 87801, USA; ccarilli@ aoc.nrao.edu \\ Received 2010 December 28; accepted 2011 February 22; published 2011 March 9
}

\begin{abstract}
I present a simple calculation of the expected mean CO brightness temperature from the large-scale distribution of galaxies during cosmic reionization. The calculation is based on the cosmic star formation rate density required to reionize, and keep ionized, the intergalactic medium, and uses standard relationships between star formation rate, IR luminosity, and $\mathrm{CO}$ luminosity derived for star-forming galaxies over a wide range in redshift. I find that the mean $\mathrm{CO}$ brightness temperature resulting from the galaxies that could reionize the universe at $z=8$ is $T_{B} \sim 1.1(C / 5)\left(f_{\text {esc }} / 0.1\right)^{-1} \mu \mathrm{K}$, where $f_{\text {esc }}$ is the escape fraction of ionizing photons from the first galaxies and $C$ is the IGM clumping factor. Intensity mapping of the $\mathrm{CO}$ emission from the large-scale structure of the star-forming galaxies during cosmic reionization on scales of order $10^{2}$ to $10^{3} \mathrm{deg}^{2}$, in combination with $\mathrm{H}$ I $21 \mathrm{~cm}$ imaging of the neutral IGM, will provide a comprehensive study of the earliest epoch of galaxy formation.
\end{abstract}

Key words: cosmology: theory - dark ages, reionization, first stars - galaxies: formation - galaxies: high-redshift - ISM: molecules - large-scale structure of universe

\section{INTRODUCTION}

Cosmic reionization corresponds to the epoch when the neutral intergalactic medium (IGM) is reionized by light from the first galaxies (Loeb \& Barkana 2001). This epoch and the preceding "dark ages" between recombination and reionization represent the "Universum Incognito"- - the last unexplored epoch of cosmic structure formation. Current observational constraints, based primarily on large-scale polarization of the cosmic microwave background (CMB) and Ly $\alpha$ scattering of light from $z>6$ quasars (the Gunn-Peterson effect), suggest that reionization may have had a large variance in space and time, starting as early as $z \sim 15$ and extending down to $z \sim 7$ (Fan et al. 2006). Interestingly, a recent census of star formation in the first galaxies suggests that, if reionization is due to the galaxies that are seen in current deep near-IR fields (with modest extrapolation to lower luminosity; e.g., Bouwens et al. 2010), the dominant epoch of reionization could occur at $z<9$ (Robertson et al. 2010).

The most direct method for studying the evolution of the neutral IGM is through the $21 \mathrm{~cm}$ line of neutral hydrogen. Extensive theoretical and observational work is ongoing to study the H I $21 \mathrm{~cm}$ signal from the neutral IGM during cosmic reionization into the preceding dark ages (Furlanetto et al. 2006; Morales \& Wyithe 2010).

An equally important, and complementary, area of study will be to trace out the large-scale structure of the star-forming galaxies that reionize the universe. Indeed, a cross correlation between the large-scale structure in star-forming galaxies, and the structure of the neutral IGM, will provide a comprehensive view of universal evolution during the epoch of first light. Relative to each other, these two probes provide "inverse views" of the universe (Lidz et al. 2009).

The difficulty in determining the galaxy distribution is the very large scales involved. The H I $21 \mathrm{~cm}$ studies are probing scales ranging from $100 \mathrm{deg}^{2}$ to $1000 \mathrm{deg}^{2}$, with depths of $z \sim$ 6-10 (Parsons et al. 2010; Lonsdale et al. 2009; Harker et al. 2010). Covering such large volumes with near-IR surveys of $z>7$ galaxies is well beyond any instrumentation in the foreseeable future. Moreover, studies of rest-frame UV light and/or the Ly $\alpha$ emission from galaxies well into reionization likely provide a biased view of the galaxy distribution due to the combination of strong Ly $\alpha$ absorption by the neutral IGM, and any dust in the host galaxies (Fontana et al. 2010).

In this Letter, I consider the possibility of tracing the largescale structure of star-forming galaxies during reionization using the technique of intensity mapping of the molecular gas in star-forming galaxies, as traced via the $\mathrm{CO}$ emission lines. Intensity mapping involves imaging the aggregate emission from thousands (or more) of galaxies on very large scales (hundreds of comoving Mpc). Individual galaxies are not detected, just the summed signal from the large-scale distribution of galaxies. This technique has been explored using the $\mathrm{H}_{\mathrm{I}} 21 \mathrm{~cm}$ line out to $z \sim 1$ (Chang et al. 2010). Note that the ALMA and the EVLA are able to detect the $\mathrm{CO}$ emission from individual, massive galaxies beyond $z \sim 6$ (Wang et al. 2010; Carilli 2010; Walter et al. 2007); however, the field of view of these instruments is small $\left(<1^{\prime}\right)$, precluding surveys on scales of tens of degrees. Intensity mapping offers a straightforward alternative for tracing the very large structure in the galaxies that reionize the neutral IGM, using smaller area telescopes, without the need to detect galaxies individually.

My goal is to estimate the mean brightness of the $\mathrm{CO}$ emission on large scales from the galaxies that reionize the neutral IGM. The notion that molecular line emission from galaxies at $z=$ $0-10$ could be a substantial CMB foreground was considered by Righi et al. (2008) using an extended Press-Schecter formalism for merging halos to set the evolution of galaxies, with analytic recipes for the implied $\mathrm{CO}$ emission from these galaxies. In this Letter, I take a simpler approach, based on the required cosmic star formation rate density to reionize the universe. This approach allows for a straightforward derivation of the mean $\mathrm{CO}$ surface brightness from the galaxies that could reionize the neutral IGM at a given redshift, although it does not predict the structure in this emission. Clearly, the uncertainties in the assumptions are significant, and these are pointed out below. However, the estimate is meant to be order of magnitude and useful for initial consideration of future instrumentation to perform $\mathrm{CO}$ intensity mapping. 


\section{CALCULATION}

Following is a calculation of the mean $\mathrm{CO}$ brightness expected from the galaxies that could reionize the universe at a given redshift. I adopt the standard concordance cosmology (Spergel et al. 2007), and comoving coordinates are used throughout. The basic information that goes into the calculation can be summarized as follows.

1. The cosmic star formation rate density required to reionize (and keep ionized) the neutral IGM.

2. A conversion from star formation rate to IR luminosity based on known properties of galaxies.

3. A conversion from IR luminosity to $\mathrm{CO}$ luminosity based on known properties of galaxies, at least out to $z \sim 3$.

\subsection{Star Formation Rate Density and IR Luminosities}

Madau et al. (1999) calculate the star formation rate density ( $\dot{\rho}_{\mathrm{SFR}}$ in $M_{\odot} \mathrm{yr}^{-1} \mathrm{Mpc}^{-3}$, comoving) required to reionize the IGM. The basic assumptions are the mean baryon density, the clumping factor (recombinations), the escape fraction of ionizing radiation from galaxies, and a Salpeter initial mass function over $0.1-100 M_{\odot}$ range. The Madau et al. calculation has been updated to the latest cosmological parameters by Bunker et al. (2010) in their Section 3.3. This relation is key to the calculation below, and I repeat it here:

$$
\begin{aligned}
\dot{\rho}_{\mathrm{SFR}}^{\text {reion }} \equiv \mathrm{SFR} / \text { Volume }= & 0.005 f_{\mathrm{esc}}^{-1}\left[\frac{(1+z)}{8}\right]^{3} \\
& \times(C / 5) X M_{\odot} \mathrm{yr}^{-1} \mathrm{Mpc}^{-3},
\end{aligned}
$$

where $f_{\text {esc }}$ is the ionizing photon escape fraction, $C$ is the clumping factor, and $X$ is a factor that depends on the cosmic baryon density and $h_{o}$, and equals 1 for the standard cosmology ( $X$ will be removed from hereon). Simulations suggest a value of $C$ somewhere between 5 and 30 (Furlanetto et al. 2006). The value of $f_{\text {esc }} \sim 0.06$ for the Milky Way and other nearby star-forming galaxies (Putman et al. 2003). Shapley et al. (2006) show that this may rise to $0.1-0.2$ for $z \sim 3$ Lyman Break Galaxies (see also Nestor et al. 2011), and there is marginal evidence that this may increase further to higher redshift (Bouwens et al. 2009).

The relationship between far-IR luminosity (FIR) and star formation rate has been considered in numerous studies over the last two decades. I adopt the standard relationship for nearby galaxies given in Kennicutt (1998):

$$
L_{\mathrm{FIR}}=1.1 \times 10^{10} \mathrm{SFR} L_{\odot},
$$

where SFR is in $M_{\odot} \mathrm{yr}^{-1}$. I will assume in the following calculation that this relationship applies on average to all galaxies, and hence applies to the volumetric average over galaxies on large scales.

In this case, the SFR as a function of $L_{\mathrm{FIR}}$ can be solved for in Equation (2), and substituted into Equation (1), yielding the FIR luminosity per unit cosmic volume required to reionize the neutral IGM:

$$
\rho_{\mathrm{FIR}}=5.5 \times 10^{7} f_{\mathrm{esc}}^{-1}\left[\frac{(1+z)}{8}\right]^{3}(C / 5) L_{\odot} \mathrm{Mpc}^{-3},
$$

where $\rho$ in this and subsequent equations indicates the comoving volume density of the subscripted quantity, in this case the FIR luminosity.

\subsection{Cosmological Relationships for $C O$ and FIR Luminosities}

The "integrated Kennicutt-Schmidt law" relates the FIR luminosity of galaxies to the CO luminosity, and also has been a topic of extensive study for both nearby and distant galaxies. The underlying physical relationship is between the star formation rate and the gas mass, but for the purposes of this Letter, the critical component is the observational relationship.

In this Letter, I employ the latest empirical relationships between CO and FIR luminosities derived by Daddi et al. (2010) for both nearby and distant star-forming galaxies. They conclude that there are two, roughly linear, relationships between $\mathrm{CO}$ luminosity and FIR luminosity for galaxies: one relevant for normal star-forming galaxies at both low and high redshifts, including the sBzK galaxies at $z \sim 2$, and the other relevant for dense starburst galaxies, such as the ULIRGs nearby, and the submillimeter galaxies and quasar hosts in the distant universe. I will use the median ratio they derive for the dominant, gas-rich, star-forming disk galaxy population at low and high redshifts:

$$
L_{\mathrm{CO}}^{\prime}=0.02 L_{\mathrm{FIR}} \mathrm{K} \mathrm{km} \mathrm{s}^{-1} \mathrm{pc}^{2} \text {, }
$$

where $L_{\text {FIR }}$ is in $L_{\odot}$.

The units for $L_{\mathrm{CO}}^{\prime}$ were originally designed for spatially resolving observations of $\mathrm{CO}$ in the Galaxy, where brightness temperature was paramount, and from which the empirical relationships between molecular gas mass $\left(\mathrm{H}_{2}\right)$ and $\mathrm{CO}$ luminosity have been calibrated. I return to this point below.

We next consider the standard relationships between $\mathrm{CO}$ luminosity and observed flux from Solomon \& Vanden Bout (2006). Since these relations are key to the calculation, I repeat the critical relations here.

Solomon \& Vanden Bout (2006) relate CO luminosity to flux density and line width as follows:

$$
L_{\mathrm{CO}}=1.0 \times 10^{3} S \Delta V(1+z)^{-1} v_{r} D_{L}^{2} L_{\odot},
$$

where the luminosity distance, $D_{L}$, is in Gpc, $v_{r}$ is the rest frequency in $\mathrm{GHz}$, flux density, $S$, is in Jy, and velocity width, $\Delta V$, is in $\mathrm{km} \mathrm{s}^{-1}$. They also give a related equation for $L_{\mathrm{CO}}^{\prime}$ :

$$
L_{\mathrm{CO}}^{\prime}=3.3 \times 10^{13} S \Delta V D_{L}^{2} \nu_{o}^{-2}(1+z)^{-3} \mathrm{~K} \mathrm{~km} \mathrm{~s}^{-1} \mathrm{pc}^{2},
$$

where $v_{o}$ is the observing frequency in GHz. Solving for $S \Delta V$ in Equations (5) and (6), and equating, yields

$$
L_{\mathrm{CO}}=3 \times 10^{-11} v_{r}^{3} L_{\mathrm{CO}}^{\prime} L_{\odot} .
$$

The cubic dependence on $v_{r}$ comes from the fact that $L_{\mathrm{CO}}^{\prime}$, being in $\mathrm{K}$, is independent of transition (for constant brightness temperature, $T_{B}$ ), hence the luminosity will increase as $v_{r}^{2}$ due to the definition of $T_{B}$, and another factor of $v_{r}$ due to the increased line width in $\mathrm{Hz}$ for the higher transitions, for a fixed velocity width in $\mathrm{km} \mathrm{s}^{-1}$.

Substituting for $L_{\mathrm{CO}}^{\prime}$ into Equation (7) using Equation (4) then yields

$$
L_{\mathrm{CO}}=6 \times 10^{-13} v_{r}^{3} L_{\mathrm{FIR}} L_{\odot} .
$$

\subsection{The CO Emission from Galaxies that Reionize the Universe}

Again, making the assumption that the individual galaxy relationships apply to the volumetric average of galaxies on large scales, we can combine Equations (8) and (3) to obtain the 
CO luminosity per unit volume required to reionize the neutral IGM:

$$
\rho_{L_{\mathrm{CO}}}=6.4 \times 10^{-8} v_{r}^{3} f_{\mathrm{esc}}^{-1}(1+z)^{3}(C / 5) L_{\odot} \mathrm{Mpc}^{-3} .
$$

Equation (9) is the fundamental relationship for the expected CO luminosity per comoving cosmic volume dictated by the star formation rate density required to reionize the neutral IGM, based on empirical relationships between $L_{\mathrm{CO}}, L_{\mathrm{FIR}}$, and star formation rate for star-forming galaxies at low and high redshifts.

We can go further and consider observable quantities. Equation (5) gives the relationship between observables $S$ and $\Delta V$ and the CO luminosity for a given galaxy. Again, assuming this applies to a volumetric average of galaxies (i.e., $\rho_{L_{\mathrm{CO}}}$ ) and using Equations (9) and (5), we obtain a velocity-integrated CO flux per unit comoving volume required for reionization:

$$
\rho_{S \Delta V}=6.4 \times 10^{-11} v_{r}^{2} D_{A}^{-2} f_{\mathrm{esc}}^{-1}(C / 5) \mathrm{Jy} \mathrm{km} \mathrm{s}^{-1} \mathrm{Mpc}^{-3},
$$

where $D_{A}$ is the angular diameter distance in Gpc. $D_{A} \sim$ $1.0 \pm 0.2 \mathrm{Gpc}$ for $z=6-10$.

\section{EXAMPLES}

Let us use Equation (10) in an example. Consider a survey at $5^{\prime}$ resolution at $z=8$ for the $\mathrm{CO} 2-1$ line $(230 \mathrm{GHz}$ rest frequency, $25.6 \mathrm{GHz}$ observing frequency). The angular size corresponds to $13 \mathrm{Mpc}$ (comoving), which implies a $\Delta z=0.044$ for the standard cosmological expansion. The comoving cosmic volume covered is then $2200 \mathrm{Mpc}^{3}$. Multiplying through by this volume, Equation (10) then yields $S \Delta V=0.073\left(f_{\text {esc }} / 0.1\right)^{-1}(C / 5)$ Jy $\mathrm{km} \mathrm{s}^{-1}$. The $\Delta z$ due to cosmic expansion corresponds to a depth of $1500 \mathrm{~km} \mathrm{~s}^{-1}$, yielding a mean signal over the band of $S=50\left(f_{\text {esc }} / 0.1\right)^{-1}(C / 5) \mu \mathrm{Jy}$.

We can then use the standard Rayleigh-Jeans relationship to derive observed brightness temperature: $T_{B}=1360 S \lambda^{2} \theta^{-2} \mathrm{~K}$, where $\theta$ is the angular size in arcseconds, $S$ is the flux density in Jy, and $\lambda^{2}$ is the observing wavelength in $\mathrm{cm}$. For a $50 \mu \mathrm{Jy}$ signal at $5^{\prime}$ resolution and observing at $1.2 \mathrm{~cm}$ wavelength: $T_{B}=1.1\left(f_{\text {esc }} / 0.1\right)^{-1}(C / 5) \mu \mathrm{K}$.

Performing the same calculation at $15^{\prime}$ resolution and $\Delta z=$ 0.13 yields $S=450\left(f_{\text {esc }} / 0.1\right)^{-1}(C / 5) \mu \mathrm{Jy}$, and again, $T_{B}=$ $1.1\left(f_{\text {esc }} / 0.1\right)^{-1}(C / 5) \mu \mathrm{K}$. Likewise, for CO $1-0$ at $15^{\prime}$ resolution, the values are $S=110\left(f_{\mathrm{esc}} / 0.1\right)^{-1}(C / 5) \mu \mathrm{Jy}$, and again, $1.1\left(f_{\mathrm{esc}} / 0.1\right)^{-1}(C / 5) \mu \mathrm{K}$.

Based on this calculation, we can estimate the expected mean $\mathrm{CO}$ brightness temperature (observed frame) as a function of redshift, for galaxies that can reionize the neutral IGM at a given redshift. The result is shown in Figure 1. Note that this is independent of transition (assuming constant $T_{B}$ for the loworder transitions) and independent of resolution (being a mean surface brightness).

\section{DISCUSSION}

I have considered the requisite $\mathrm{CO}$ emission coming from the galaxies that reionize the universe at a given redshift. The calculation above leads to a mean surface brightness at $z=8$ of

$$
T_{B} \sim 1.1\left(f_{\mathrm{esc}} / 0.1\right)^{-1}(C / 5) \mu \mathrm{K}
$$

with a gradual variation with redshift (Figure 1).

Note that real observations will entail detecting fluctuations in the CO signal around the mean. Also, this value of $T_{B}$ is

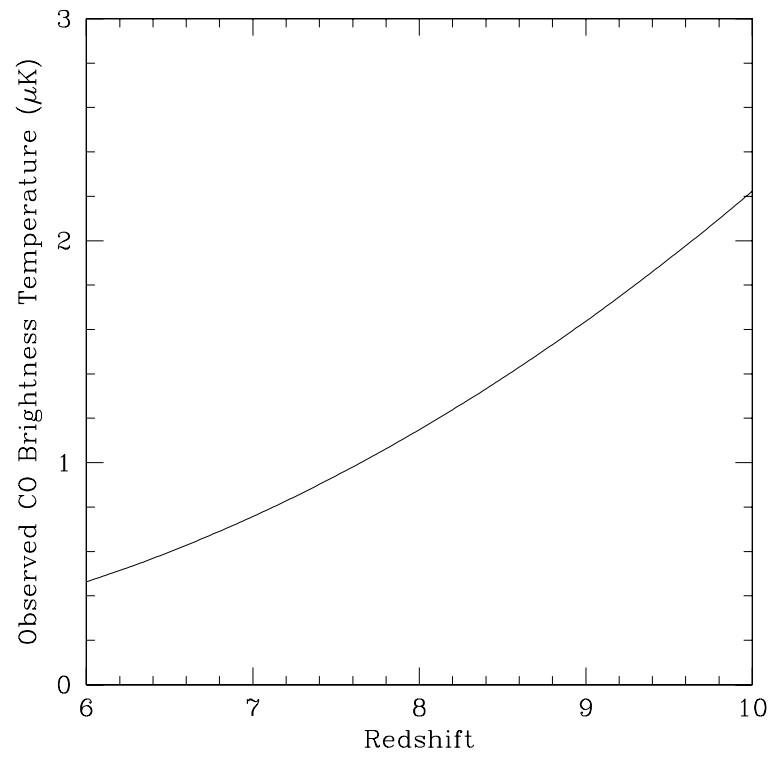

Figure 1. Expected mean $\mathrm{CO}$ brightness temperature (observed frame) for galaxies that can reionize, and keep ionized, the neutral IGM at a given redshift, based on Equation (10) and assuming $f_{\mathrm{esc}}=0.1$ and $C=5$.

dictated by the star formation rate density at a given redshift that is needed to reionize, and keep ionized, the neutral IGM (Equation (10)). In reality, the star formation rate density will evolve as it will, and we will observe over what redshift range the IGM reionizes.

There are clearly substantial uncertainties in the calculation above. Explicit uncertainties involve $f_{\text {esc }}$ and $C$, both of which have current estimates that could range by a factor of a few (see Section 2.1). Determining the escape fraction of ionizing photons will be a major goal of future observations of $z>7$ galaxies with JWST, while determining the clumping factor is clearly a goal of next generation H I $21 \mathrm{~cm}$ reionization experiments.

Implicit to the calculation are the relationships between star formation rate, FIR luminosity, and CO luminosity. The dust and $\mathrm{CO}$ production and heating in the first galaxies remains uncertain. Hence, it remains unclear whether one can extrapolate standard Kennicutt-Schmidt star formation laws, and FIR luminosity to star formation rate relations, to very early galaxies. Detection of strong $\mathrm{CO}$ line emission, and thermal emission from warm dust, from $z>6$ quasar host galaxies suggests that $\mathrm{CO}$ and dust can be produced rapidly in the first star-forming galaxies, although these systems are at the high-mass end of the galaxy distribution (Wang et al. 2008, 2010). As for CO excitation, clearly the CMB will "depopulate" the lowest levels at high $z$, although a calculation of the Boltzmann distribution suggests only a factor two or so decrease in the population of the first excited state at $z \sim 7$ in a typical star-forming galaxy, relative to having no background at all. Calibrating the relationships between CO luminosity, FIR emission, and star formation from $z>7$ galaxies will be a major goal with ALMA, the EVLA, and the JWST, in the coming years, on a galaxy-by-galaxy basis. This information can then be incorporated into the calculation above for a better estimate of the average $\mathrm{CO}$ emission seen on very large scales by intensity mapping.

Another difficulty will be line confusion. The observed line cubes will contain different transitions at different redshifts. To make full use of the data, a dual-frequency experiment may be required, probing two transitions of $\mathrm{CO}$ to eliminate ambiguities, 
although cross correlation with the H $\mathrm{H} 21 \mathrm{~cm}$ images will also help.

Overall, the estimate of $T_{B}$ for $\mathrm{CO}$ above should be considered at best accurate to an order of magnitude. We can compare this value to more involved calculations in the recent literature. Righi et al. (2008) have considered the background radiation expected from $\mathrm{CO}$ lines from galaxies from $z=1$ to 10 , in the frequency range of $\sim 10-50 \mathrm{GHz}$. Their calculation adopted the extended Press-Schecter formalism to set the galaxy merger rate and used analytic recipes for the implied $\mathrm{CO}$ emission from these galaxies. They obtain a mean signal from a given $\mathrm{CO}$ transition at $z \sim 2$ within a factor two of the mean calculated herein. More recently, Gong et al. (2011) have used the Millennium simulation results of Obreschkow et al. (2009) to estimate the CO surface brightness fluctuations on large scales at $z>6$. The Obreschkow et al. study involved generating mock galaxy catalogs in $\mathrm{CO}$ emission based on the Millennium dark matter cosmological simulation, and again, invoking recipes to extrapolate to $\mathrm{CO}$ luminosity for a given halo. Gong et al. find a mean CO surface brightness of $\sim 0.1-0.7 \mu \mathrm{K}$ at $z \sim 7$ (depending on model assumptions), with fluctuations a factor of a few lower than this on scales $\sim 10^{\prime}$ (see also S. Furlanetto et al. 2011, in preparation). The roughly factor two agreement between these sophisticated calculations and the simple estimate presented herein is encouraging, given the very different approaches.

Is it plausible to detect such a signal? Without going into detailed instrument design, we can consider receiver noise and brightness temperature (i.e., the radiometry equation). For a $20 \mathrm{~K}$ receiver system in $1000 \mathrm{hr}$ and a $130 \mathrm{MHz}$ channel $\left(=1500 \mathrm{~km} \mathrm{~s}^{-1}\right.$ at $\left.26 \mathrm{GHz}\right), \delta T=20 \mathrm{~K} /\left[3.6 \times 10^{6} \times 1.3 \times\right.$ $\left.10^{8}\right]^{1 / 2}=0.9 \mu \mathrm{K}$. Hence, achieving a $1 \sigma$ brightness sensitivity is plausible in a long integration.

A key point to keep in mind is that the initial studies will be statistical in nature, e.g., a cross correlation with the $\mathrm{H} \mathrm{I}$ $21 \mathrm{~cm}$ images, or study of the autocorrelation of the $\mathrm{CO}$ cubes themselves. The challenge will be obtaining the combination of very wide field ( $>100 \mathrm{deg}^{2}$ ) with $\mu \mathrm{K}$ surface brightness sensitivity at resolutions $\sim 1^{\prime}-10^{\prime}$, at frequencies between 15 and $45 \mathrm{GHz}$. Gong et al. (2011) consider this point in more detail. They estimate that a 1000 element array of $0.7 \mathrm{~m}$ diameter antennas with a $1 \mathrm{GHz}$ bandwidth and $30 \mathrm{MHz}$ channels operating at $15 \mathrm{GHz}$ could detect the $\mathrm{CO}$ power spectrum to high significance in $3000 \mathrm{hr}$ on comoving scales of $k \sim 0.1-1 \mathrm{Mpc}^{-1}$. J. Bowman et al. (2011, in preparation) are also considering the design for such an experiment, including the relative benefits of interferometers versus focal plane arrays on single-dish telescopes.

Ultimately, the critically unique analysis will be the cross correlation with the $\mathrm{H}_{\mathrm{I}} 21 \mathrm{~cm}$ image cubes (Gong et al. 2011), which is well beyond the scope of this Letter. My main conclusion is that the simple calculation presented herein, based on the star formation rate required to reionize the universe, supports the more sophisticated treatments of the problem, and suggests that the $\mathrm{CO}$ intensity mapping experiment at $z>6$ is plausible.

C.C. thanks the Keck Institute for Space Studies for instigating this work, the $\mathrm{CO}$ intensity mapping design team for lively discussions, and the referee for insightful comments that improved this Letter.

\section{REFERENCES}

Bouwens, R., et al. 2009, ApJ, 705, 936

Bouwens, R., et al. 2010, ApJ, 709, L133

Bunker, A., et al. 2010, MNRAS, 409, 855

Carilli, C. L. 2010, in AIP Conf. Proc. 1294, The First Stars and Galaxies: Challenges for the Next Decade, ed. D. J. Whalen, V. Bromm, \& N. Yoshida (Melville, NY: AIP), 234

Chang, T.-C., et al. 2010, Nature, 466, 463

Daddi, E., et al. 2010, ApJ, 714, L118

Fan, X., Carilli, C., \& Keating, B. 2006, ARA\&A, 44, 415

Fontana, A., et al. 2010, ApJ, 725, L205

Furlanetto, S., Oh, S. P., \& Briggs, F. 2006, Phys. Rep., 433, 181

Gong, Y., Cooray, A., Silva, M., Santos, M., \& Lubin, P. 2011, ApJ, 728, L46

Harker, G., et al. 2010, MNRAS, 405, 2492

Kennicutt, R. 1998, ARA\&A, 36, 189

Lidz, A., et al. 2009, ApJ, 690, 252

Loeb, A., \& Barkana, R. 2001, ARA\&A, 39, 19

Lonsdale, C. J., et al. 2009, Proc. IEEE, 97, 1497

Madau, P., Haardt, F., \& Rees, M. 1999, ApJ, 514, 648

Morales, M., \& Wyithe, S. 2010, ARA\&A, 48, 127

Nestor, D., Shapley, A., Steidel, C., \& Siana, B. 2011, ApJ, submitted (arXiv:1102.0286v1)

Obreschkow, D., et al. 2009, ApJ, 698, 1467

Parsons, A., et al. 2010, AJ, 139, 1468

Putman, M., et al. 2003, ApJ, 597, 948

Righi, M., Hernandez-Monteagudo, C., \& Sunyaev, R. 2008, A\&A, 489, 489

Robertson, B., et al. 2010, Nature, 468, 49

Shapley, A., et al. 2006, ApJ, 651, 688

Solomon, P., \& Vanden Bout, P. 2006, ARA\&A, 43, 677

Spergel, D., et al. 2007, ApJS, 179, 377

Walter, F., Carilli, C. L., Bertoldi, F., Cox, P., \& Menten, K. M. 2007, Highlights Astron., 14, 263

Wang, R., et al. 2008, ApJ, 687, 848

Wang, R., et al. 2010, ApJ, 714, 699 